\title{
Does intrauterine injection of low-molecular-weight heparin improve the clinical pregnancy rate in intracytoplasmic sperm injection?
}

\author{
Ahmed Mohamed Kamel ${ }^{1,2}$, Yahia El-Faissal ${ }^{1,2}$, Mona Aboulghar ${ }^{1,2}$, Ragaa Mansour ${ }^{2}$, Gamal I Serour ${ }^{2,3}$, Mohamed Aboulghar ${ }^{1,2}$ \\ ${ }^{1}$ Department of Obstetrics and Gynecology, Faculty of Medicine, Cairo University, Cairo; ${ }^{2}$ Egyptian IVF and ET Center, Cairo; ${ }^{3}$ Department of Obstetrics \\ and Gynecology, Faculty of Medicine, Al Azhar University, Cairo, Egypt
}

Objective: Heparin can modulate proteins, and influence processes involved in implantation and trophoblastic development. This study aimed to assess the improvement of clinical pregnancy and implantation rates after local intrauterine injection of low-molecular-weight heparin $(\mathrm{LMWH})$ in patients undergoing intracytoplasmic sperm injection (ICSI).

Methods: A randomised case/control design was followed in women scheduled for ICSI. The study arm was injected with intrauterine LMWH during mock embryo transfer immediately following the ovum pickup procedure, while the control arm was given an intrauterine injection with a similar volume of tissue culture media. Side effects, the clinical pregnancy rate, and the implantation rate were recorded.

Results: The pregnancy rate was acceptable (33.9\%) in the LMWH arm with no significant reported side effects, confirming the safety of the intervention. No statistically significant differences were found in the clinical pregnancy and implantation rates between both groups $(p=0.182$ and $p=0.096$, respectively). The odds ratio of being pregnant after intrauterine injection with LMWH compared to the control group was 0.572 (95\% confidence interval [Cl], 0.27-1.22), while the risk ratio was $0.717(95 \% \mathrm{Cl}, 0.46-1.13 ; p=0.146)$. No statistical significance was found between the two groups in other factors affecting implantation, such as day of transfer $(p=0.726)$, number of embryos transferred $(p=0.362)$, or embryo quality.

Conclusion: Intrauterine injection of LMWH is a safe intervention, but the dose used in this study failed to improve the outcome of ICSI. Based on its safety, further research involving modification of the dosage and/or the timing of administration could result in improved ICSI success rates.

Keywords: Embryo transfer; Intracytoplasmic sperm injection; Low-molecular-weight heparin

\section{Introduction}

Heparin is a polysulfhated glycosaminoglycan that interacts with proteins containing positively charged amino acids [1]. Low-molecular-weight heparin (LMWH) is derived from heparin by enzymatic chemical depolymerisation of unfractionated heparin [2].

Received: Aug 21, 2016 · Revised: Oct 17, 2016 · Accepted: Oct 31, 2016

Corresponding author: Ahmed Mohamed Kamel

Department of Obstetrics and Gynecology, Faculty of Medicine, Cairo University, KasrAlainy St., Garden City, Cairo 11562, Egypt

Tel:+20-1120022332 Fax:+20-225253532 E-mail:dr.ahmed.m.kamel@gmail.com

This is an Open Access article distributed under the terms of the Creative Commons Attribution Non-Commercial License (http://creativecommons.org/licenses/by-nc/4.0/) which permits unrestricted non-commercial use, distribution, and reproduction in any medium, provided the original work is properly cited.
Heparin and heparin sulphate have a high binding affinity due to their highly anionic nature. This allows them to bind to antithrombin, growth factors and their receptors, viral envelope proteins, and extracellular matrix molecules. Heparin sulphate proteoglycans are also expressed throughout the genital tract, and are involved in the regulation of endometrial cycling [3].

The ability of heparin to bind with and modulate a wide variety of proteins can influence a number of physiological processes involved in implantation and trophoblastic development. These processes include adhesion of the blastocyst to the endometrial surface, trophoblastic differentiation, and invasion [4].

In patients with inherited and acquired thrombophilia in early pregnancy, the traditional role for LMWH was in the prevention of 
clotting during implantation and placentation [5]. Unfractionated heparin as well as LMWH can positively modulate the process of decidualisation, and therefore can improve implantation in assisted reproductive techniques (ART) [6].

A recent Cochrane review showed no added value for the use of systemic heparin in in vitro fertilization (IVF) cycles; however, they proposed studying the possible effects of the local (uterine) application of heparin during ART [7].

Based on the above evidence, we formulated the hypothesis that the intrauterine injection of LMWH might help improve implantation and clinical pregnancy rates in patients undergoing ART. We studied the effect of local intrauterine LMWH injection on clinical pregnancy and implantation rates and confirmed its safety by using it locally without compromising pregnancy.

\section{Methods}

\section{Study design}

The study was approved by the Internal Review Board of the Egyptian IVF and ET Center (protocol ID: 152014) on October 12, 2014, and later registered at https://clinicaltrials.gov with the identifier NCT02325479. All patients who agreed to join the study provided written informed consent and agreed to let us use the gathered data for publication.

Consecutive patients undergoing an ovum pick-up (OPU) procedure and embryo transfer (ET) at the Egyptian IVF Center were recruited for this randomized controlled study. The inclusion criteria were age between 20 and 37 years, normal serum prolactin and thyroid-stimulating hormone levels, unexplained or tubal factor infertility, body mass index (BMl) $\leq 30 \mathrm{~kg} / \mathrm{m}^{2}$, and a normal coagulation profile. The patients were also screened for inherited and acquired thrombophilias. The exclusion criteria included patients with reduced ovarian reserve diagnosed by a low antral follicle count (AFC) or elevated follicle-stimulating hormone (FSH) and luteinizing hormone (LH) levels, frozen-thawed ET cycles, uterine anomalies, submucous fibroids and polyps, uterine synechia, and any contraindication to pregnancy.

The patients were divided into two groups. The treatment group was injected with intrauterine LMWH (enoxaparin sodium; Clexane, Sanofi, Paris, France) during mock ET, just after OPU ( $2-5$ days prior to ET), while the control group was injected with a similar volume of tissue culture media (G.2 plus ref. 10132; Vitrolife AB, Göteborg, Sweden) instead of $L M W H$.

\section{Randomization and sample size calculation}

In order to ensure the safety of the intervention performed in this study, the Internal Review Board agreed to only enroll 20 patients ini- tially in each study arm in a pilot study to determine if the drug had any harmful side effects or would severely compromise the pregnancy rate. A third party (managerial secretary) not involved in the study was assigned to record the results to prevent performance bias on the part of the staff involved. An agreement was also made to stop the study if more than 10 consecutive patients in the intervention group (intrauterine LMWH) failed to get pregnant. The data retrieved from this pilot study was then to be used to calculate a sample size with appropriate power for a randomised controlled trial.

According to the results of our pilot study, the clinical pregnancy rate in the LMWH group was $56 \%$, while in the control group it was $30 \%$; we set the a error level at 0.5 and the power at $80 \%$. Fifty-three subjects were needed in each study arm with a 1:1 ratio. We allowed for a drop-out rate of $10 \%$ to $12 \%$, and therefore decided to recruit 60 women into each group.

Patients were assigned into one of the two groups using a computer-generated randomisation table. Allocation into either group was done through by operating room nurse, who selected a closed opaque envelope containing the designation. The laboratory staff prepared the study drugs in identical syringes, and stored them separately beforehand. All patients, doctors, nurses, and laboratory staff were blinded to which cases were in the intervention arm.

\section{Dose calculation}

The dose of LMWH given was calculated according to a previous study by our group [8], in which the intrauterine administration of $500 \mathrm{IU}$ of human chorionic gonadotrophin (hCG) improved the implantation and pregnancy rates.

Since this had not been attempted before, the best way to compare two different drugs was through biological activity. Therefore, since we wanted to use $500 \mathrm{IU}$ of LMWH and knew that in the in vitro purified system, enoxaparin sodium has a high anti-activated StuartPrower factor (anti-Xa) activity (approximately $100 \mathrm{IJ} / \mathrm{mg}$ ), $5 \mathrm{mg}$ was necessary to obtain $500 \mathrm{IU}$ of LMWH. A $0.2-\mathrm{mL}$ syringe contains 20 $\mathrm{mg}$ of Clexane, and therefore we needed to perform an intrauterine injection of $0.05 \mathrm{~mL}$ a using a Labotect ET catheter (Labotect, Göttingen, Germany).

\section{Intracytoplasmic sperm injection}

The standard long gonadotrophin-releasing hormone agonist protocol was used for all patients. Oocytes were then fertilized by intracytoplasmic sperm injection (ICSI) as described elsewhere [9], and ET was performed 2 to 5 days later. Progesterone was administered for luteal phase support using daily intramuscular injections of $100 \mathrm{mg}$ of Prontogest (IBSA, Institut Biochimique SA, Lugano, Switzerland) or 400-mg pessaries twice daily (Cyclogest 400 mg, Actavis plc, Dublin, Ireland). Serum $\beta$-hCG was assessed 2 weeks after ET, and clinical 
pregnancy was confirmed by transvaginal ultrasonography (TV-US) for confirmation of cardiac activity at 6 weeks' gestation. Pregnant patients were followed up until 12 weeks of pregnancy to determine the early miscarriage rate.

\section{Study outcomes and statistical analysis}

The primary outcomes included clinical pregnancy, implantation, and miscarriage rates. The secondary outcome of the study was to assess the safety of locally applying LMWH before ET by determining how it affected the clinical pregnancy rate and recording side effects of the treatment.

The data were statistically described as mean \pm standard deviation or frequencies and percentages when appropriate. The chi-square test was used to compare categorical data and the odds ratio (OR). Numerical variables were compared between the study groups using the Student's $t$-test or the Mann-Whitney $U$ test for independent samples as appropriate. Two-tailed $p$-values $<0.05$ were considered to indicate statistical significance. All statistical calculations were done using SPSS ver. 15.0 for Windows (SPSS Inc., Chicago, IL, USA). The sample size was calculated using an online calculator (http:// powerandsamplesize.com/Calculators) using the formula for two proportions with two-sided equality.
The trial was registered at clinicaltrials.gov with the identifier NCT02325479. Recruitment started in January 2015.

\section{Results}

Between January 2015 and December 2015, 180 patients were assessed for eligibility (Figure 1). A total of 120 patients were ultimately recruited for the study. Six patients were excluded because ET had not been performed due to their increased risk of ovarian hyperstimulation syndrome, and their embryos were therefore frozen. No patients were lost to follow-up, and we ultimately enrolled 59 women in the LMWH group and 55 controls.

The baseline characteristics of the patients included in our study are shown in Table 1. No significant differences were found between the women with regard to age ( $p=0.488)$, type $(p=0.719)$, and duration of infertility $(p=0.567)$; $\mathrm{BMI}(p=0.408) ; \mathrm{FSH}(p=0.633)$; LH $(p=0.835)$; and AFC $(p=0.113)$.

Table 2 shows a clinical pregnancy rate of 33.9\% in the LMWH group compared to a rate of $47.2 \%$ in the control group ( $p=0.182$ ). The implantation rate was also lower in the LMWH group (18.2\%) than in the control group (22.3\%) $(p=0.096)$. First trimester miscarriages were $0.3 \%$ less common in the $\mathrm{LMWH}$ group than in the con-

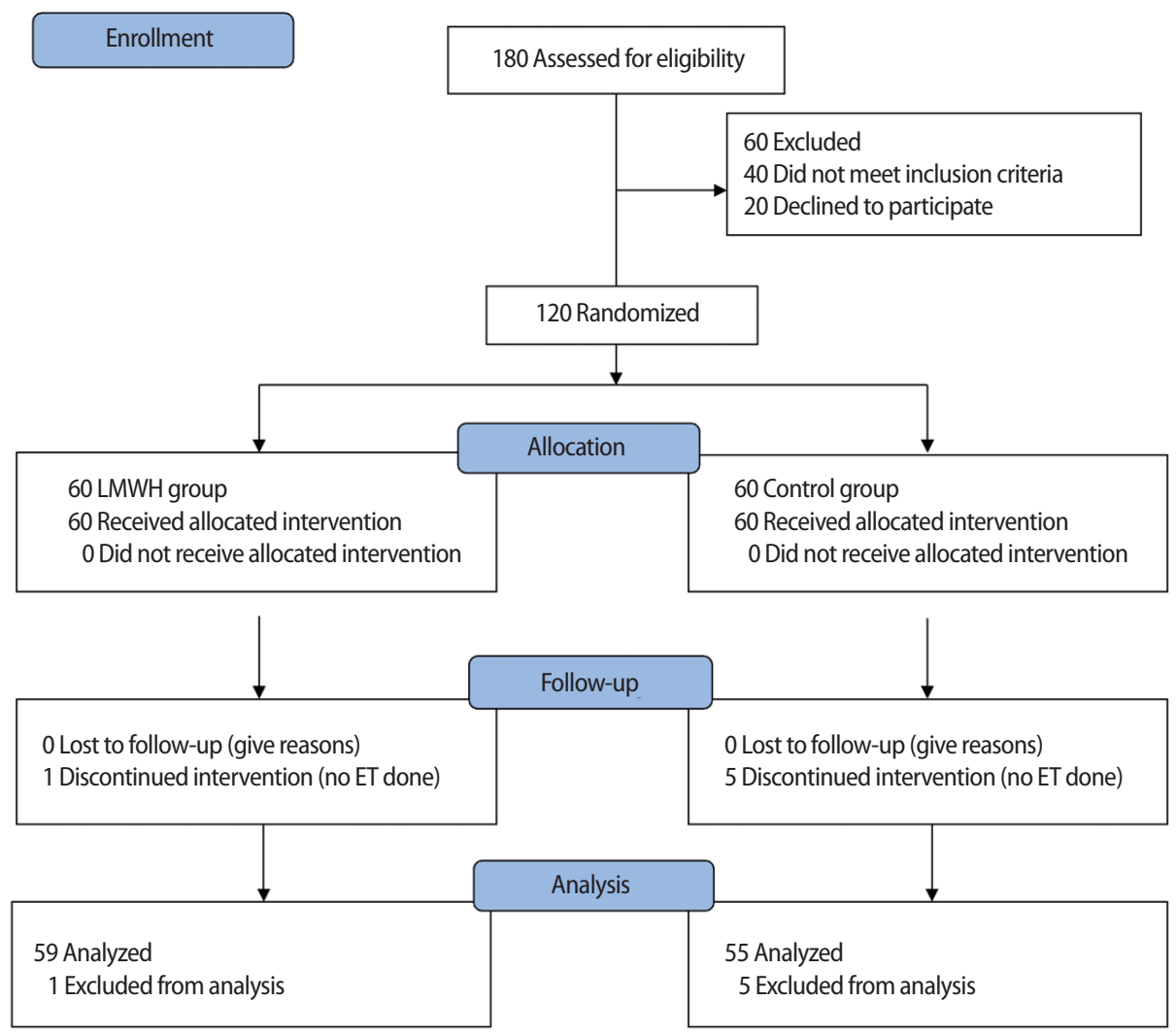

Figure 1. Enrollment flow chart. LMWH, low-molecular-weight heparin; ET, embryo transfer. 
Table 1. Comparison of clinical characteristics between women who underwent a local LMWH injection and controls

\begin{tabular}{lccc}
\hline Variable & LMWH group & Control group & $p$-value \\
\hline No. of patients & 59 & 55 & 0.488 \\
Age $(\mathrm{yr})$ & $28.80 \pm 5.17(27.45-30.14)$ & $28.15 \pm 4.79(26.85-29.44)$ & $16(29.1)$ \\
Primary infertility & $19(32.2)$ & $5.24 \pm 2.58(4.54-5.93)$ & 0.719 \\
Duration of infertility $(\mathrm{yr})$ & $5.54 \pm 3.12(4.73-6.36)$ & $27.41 \pm 2.30(26.79-28.04)$ & 0.567 \\
BMl $\left(\mathrm{kg} / \mathrm{m}^{2}\right)$ & $27.07 \pm 2.15(26.51-27.63)$ & $5.27 \pm 2.41(4.62-5.92)$ & 0.408 \\
FSH $(\mathrm{IU} / \mathrm{mL})$ & $5.06 \pm 2.35(4.48-5.67)$ & $5.02 \pm 2.11(4.45-5.60)$ & 0.633 \\
LH $(\mathrm{IU} / \mathrm{mL})$ & $4.94 \pm 2.29(4.34-5.53)$ & $17.90 \pm 3.60(16.93-18.88)$ & 0.835 \\
Antral follicle count $(\mathrm{n})$ & $16.84 \pm 3.50(15.93-17.76)$ & 0.113 \\
\hline
\end{tabular}

Values are presented as mean \pm standard deviation ( $95 \%$ confidence interval) or number (\%).

The Student'st-test was used for all parameters, except primary infertility, which was tested using the chi-square test.

LMWH, low-molecular-weight heparin; BMI, body mass index; FSH, follicle-stimulating hormone; LH, luteinizing hormone.

Table 2. Comparison of clinical outcomes in women who underwent a local LMWH injection and controls

\begin{tabular}{lccc}
\hline Variable & LMWH group & Control group & $p$-value \\
\hline No. of patients & 59 & 55 & 0.182 \\
Clinical pregnancy & $20(33.9)$ & $26(47.2)$ & 0.627 \\
Miscarriage & $3(15)$ & $4(15.3)$ & 0.096 \\
Implantation rate (\%) & 18.2 & 22.3 & 0.146 \\
Odds ratio for pregnancy & $0.572(0.27-1.22)$ & $1.75(0.82-3.72)$ & 0.146 \\
Relative risk for pregnancy & $0.717(0.46-1.13)$ & $1.254(0.92-1.70)$ & \\
\hline
\end{tabular}

Values are presented as number (\%) or odds ratio (95\% confidence interval) unless otherwise indicated.

The chi-square test was used to compare categorical data and ratios.

$\mathrm{LMWH}$, low-molecular-weight heparin.

Table 3. Comparison of factors affecting implantation, embryo quality, and embryo transfer between the study groups

\begin{tabular}{lccc}
\hline Variable & LMWH group & Control group & $p$-value \\
\hline No. of patients & 59 & 55 & $15(27.3)$ \\
Severe male factor infertility & $18(30.5)$ & $5(9.1)$ & 0.703 \\
Recurrent implantation failure & $7(11.9)$ & $13.60 \pm 5.23(12.18-18.01)$ & 0.630 \\
No. of oocytes retrieved & $14.17 \pm 5.39(12.76-15.58)$ & $4.01 \pm 0.95(3.76-4.28)$ & 0.609 \\
Day of transfer & $4.10 \pm 0.86(3.88-4.33)$ & $2.52 \pm 0.66(2.34-2.71)$ & 0.726 \\
No. of embryos transferred & $2.42 \pm 0.62(2.26-2.59)$ & $64 / 282(22.7)$ & 0.362 \\
Grade l embryos transferred & $66 / 282(23.4)$ & $60 / 282(21.3)$ & 0.984 \\
Grade Il embryos transferred & $61 / 282(21.6)$ & $15 / 282(5.3)$ & 0.577 \\
Grade III embryos transferred & $16 / 282(5.6)$ & 0.476 & \\
\hline
\end{tabular}

Values are presented as number (\%) or mean \pm standard deviation (95\% confidence interval).

The Mann-Whitney $\mathrm{U}$ test was used to compare numerical data; the chi-square test was used to compare categorical data and ratios.

$\mathrm{LMWH}$, low-molecular-weight heparin.

trol group ( $p=0.627)$, but none of these differences were statistically significant. All cases of first-trimester miscarriages were diagnosed as missed miscarriages, and all occurred within the first 9 weeks of gestation. Only two cases in the control group had foetal cardiac pulsation visualised at 7 weeks, before later stopping at follow-up TV-US at 9 weeks of gestation.

Having a positive pregnancy test with an intrauterine injection of LMWH had an OR of 0.572 (95\% confidence interval [Cl], 0.27-1.22) compared to the control group $(\mathrm{OR}, 1.75 ; 95 \% \mathrm{Cl}, 0.82-3.72)$. This shows that the odds of becoming pregnant were lower if the patient had a local LMWH injection, but the results were statistically non-significant $(p=0.146)$.

Some of the factors affecting implantation and pregnancy relating to ICSI are presented in Table 3. The day of transfer $(p=0.726)$, number of embryos transferred $(p=0.362)$, and embryo quality showed no statistically significant difference between the two groups. Likewise, no statistically significant differences were found in patients with recurrent implantation failure ( $>3$ previous trials) or cases 
where severe male factor infertility (azoospermia or severe astheno/ teratozoospermia) was the indication for ICSI ( $p=0.630$ and $p=0.703$, respectively).

No side effects associated with LMWH overdose, such as purpuric patches or rashes, excessive bruising, or nose bleeds, were reported in the patients in either group. Twenty patients complained of bleeding during the luteal support phase, only six of whom belonged to the LMWH group.

\section{Discussion}

In spite of advances in long-standing ART procedures, the implantation rate remains between $10 \%$ and $30 \%$, and pregnancy rates are still as low as $28.3 \%$ to $44.5 \%$ [10].

Researchers are looking for techniques and treatment modalities to improve these rates. Subcutaneous heparin from the day of ET is a modality that is being used more often, especially in patients with recurrent implantation failure; however, it is not supported by sufficient evidence [11]. A recently published Cochrane review showed no statistically significant advantage of adding systemic heparin after ET, but suggested that local intrauterine heparin might play a role [7]. To the best of our knowledge, we are the first group to study the effects of intrauterine LMWH on the clinical pregnancy rate.

Research done by Urman et al. [12] showed that in patients with no proven thrombophilic disorders, heparin improved implantation. Evidence now suggests that heparin exerts this beneficial effect by modulating endometrial receptivity and decidualising the endometrial stroma. Heparin modulates heparin-binding epidermal growth factor, which assists in the process of implantation, trophoblastic invasion, and early embryo development [13-15]. The production of endometrial prolactin and insulin-like growth factor by the endometrium is increased, while levels of insulin-like growth-factor-binding protein decrease under the influence of heparin [6]. These factors play a crucial role in endometrial maturity and optimizing receptivity during the implantation window [16-18].

In this study, we found that local intrauterine LMWH injections did not decrease the pregnancy rate below known acceptable standards [10]; however, the clinical pregnancy rate among the controls was higher to a non-statistically significant extent. This proves that the occurrence of pregnancy was not adversely affected by this intervention, and since there were no serious reported side effects attributed to this treatment modality, it is considered safe. However, the mere fact that it is a safe intervention does not justify its further use with the same dosage and timing of administration, since it failed to increase pregnancy and implantation rates when compared to the control group.

Akhtar et al. [7] reported a higher OR than we found $(1.66 ; 95 \% \mathrm{Cl}$,
0.94-2.9) for pregnancy when systemic heparin was given in the peri-implantation period to improve clinical outcomes in women undergoing ART, but those results also proved non-significant. In 2013, another meta-analysis by Potdar et al. [11] showed statistically significant improvement only in the live birth rate (relative risk, 0.22; 95\% $\mathrm{Cl}, 0.06-0.78 ; p=0.02$ ) with systemic LMWH. They also found no significant improvement in the implantation rate.

In 2013, Fawzy and El-Refaeey [19] studied the effect of combining prednisolone ( $20 \mathrm{mg} /$ day) with LMWH ( $1 \mathrm{mg} / \mathrm{kg} /$ day) daily starting from the day of oocyte pick-up in patients with recurrent implantation failure, and found a significant increase in both clinical pregnancy and implantation rates; the clinical pregnancy rate was $40.7 \%$ in the treatment group versus $27.5 \%$ in the control group, and the implantation rates were $23.9 \%$ and $14.7 \%$, respectively.

Urman et al. [12], Qublan et al. [20], and Berker et al. [21] have shown a statistically significant reduction in the miscarriage rate in their intervention groups receiving heparin, whereas our results agree with those of Potdar et al. [11], who found no statistically significant effect on the miscarriage rate.

Limitations to our work include the calculation of the optimal dose for the experiment, because we had no previous data to rely on, and so little work has been published on the intrauterine injection of chemicals as adjuvants for ART. We therefore relied on previous work by Mansour et al. [8], in which we calculated the same dose in IU for both compounds using their biological activity. Choosing the optimal time for the intervention also proved challenging. It was impossible to foresee the effect the LMWH would have on live embryos in culture. Therefore, we made the ethical decision to inject it after OPU, as all the desired effects on the endometrium could be achieved before ET, without placing the transferred embryos at risk of being negatively affected by an untested compound in tissue culture. Although the injection of LMWH preceded ET by 3 to 5 days, previously described changes occurring in the endometrium from exposure to LMWH $[4,6,13-18]$ should persist till the day of transfer, because such changes to the endometrium are irreversible.

The local intrauterine injection of LMWH using the above-described dose and timing proved safe, but showed no benefit and no increase in either implantation or pregnancy rates in ICSI patients. Insufficient evidence still exists for the possibility that systemic or local LMWH injections could improve the outcome of ICSI cycles. However, more research is needed to assess the dosage and timing of local administration and the value of adding LMWH to culture media.

\section{Conflict of interest}

No potential conflict of interest relevant to this article was reported. 


\section{References}

1. Linhardt RJ, Wang HM, Ampofo SA. New methodologies in heparin structure analysis and the generation of LMW heparins. Adv Exp Med Biol 1992;313:37-47.

2. Xu X, Ding J, Rao G, Shen J, Prinz RA, Rana N, et al. Estradiol induces heparanase-1 expression and heparan sulphate proteoglycan degradation in human endometrium. Hum Reprod 2007; 22:927-37.

3. Germeyer A, Klinkert MS, Huppertz AG, Clausmeyer S, Popovici RM, Strowitzki T, et al. Expression of syndecans, cell-cell interaction regulating heparan sulfate proteoglycans, within the human endometrium and their regulation throughout the menstrual cycle. Fertil Steril 2007;87:657-63.

4. Nelson SM, Greer IA. The potential role of heparin in assisted conception. Hum Reprod Update 2008;14:623-45.

5. Poehlmann TG, Fitzgerald JS, Meissner A, Wengenmayer T, Schleussner $\mathrm{E}$, Friedrich $\mathrm{K}$, et al. Trophoblast invasion: tuning through LIF, signalling via Stat3. Placenta 2005;26 Suppl A:S37-41.

6. Fluhr H, Spratte J, Ehrhardt J, Steinmuller F, Licht P, Zygmunt M. Heparin and low-molecular-weight heparins modulate the decidualization of human endometrial stromal cells. Fertil Steril 2010; 93:2581-7.

7. Akhtar MA, Sur S, Raine-Fenning N, Jayaprakasan K, Thornton J, Quenby $S$, et al. Heparin for assisted reproduction: summary of a Cochrane review. Fertil Steril 2015;103:33-4.

8. Mansour R, Tawab N, Kamal O, El-Faissal Y, Serour A, Aboulghar M, et al. Intrauterine injection of human chorionic gonadotropin before embryo transfer significantly improves the implantation and pregnancy rates in in vitro fertilization/intracytoplasmic sperm injection: a prospective randomized study. Fertil Steril 2011;96:1370-4.e1.

9. Mansour R, Fahmy I, Tawab NA, Kamal A, El-Demery Y, Aboulghar $M$, et al. Electrical activation of oocytes after intracytoplasmic sperm injection: a controlled randomized study. Fertil Steril 2009; 91:133-9.

10. Baruffi RL, Mauri AL, Petersen CG, Nicoletti A, Pontes A, Oliveira $J B$, et al. Single-embryo transfer reduces clinical pregnancy rates and live births in fresh IVF and intracytoplasmic sperm injection (ICSI) cycles: a meta-analysis. Reprod Biol Endocrinol 2009;7:36.

11. Potdar N, Gelbaya TA, Konje JC, Nardo LG. Adjunct low-molecu- lar-weight heparin to improve live birth rate after recurrent implantation failure: a systematic review and meta-analysis. Hum Reprod Update 2013;19:674-84.

12. Urman B, Ata B, Yakin K, Alatas C, Aksoy S, Mercan R, et al. Luteal phase empirical low molecular weight heparin administration in patients with failed ICSI embryo transfer cycles: a randomized open-labeled pilot trial. Hum Reprod 2009;24:1640-7.

13. Tamada H, Higashiyama C, Takano H, Kawate N, Inaba T, Sawada T. The effects of heparin-binding epidermal growth factor-like growth factor on preimplantation-embryo development and implantation in the rat. Life Sci 1999;64:1967-73.

14. Constancia M, Hemberger M, Hughes J, Dean W, FergusonSmith A, Fundele R, et al. Placental-specific IGF-II is a major modulator of placental and fetal growth. Nature 2002;417:9458.

15. Stevenson JL, Choi SH, Varki A. Differential metastasis inhibition by clinically relevant levels of heparins: correlation with selectin inhibition, not antithrombotic activity. Clin Cancer Res 2005; 11(19 Pt 1):7003-11.

16. Wilcox AJ, Baird DD, Weinberg CR. Time of implantation of the conceptus and loss of pregnancy. N Engl J Med 1999;340:17969.

17. Fluhr H, Krenzer S, Stein GM, Stork B, Deperschmidt M, Wallwiener $D$, et al. Interferon-gamma and tumor necrosis factor-alpha sensitize primarily resistant human endometrial stromal cells to Fas-mediated apoptosis. J Cell Sci 2007;120:4126-33.

18. Fluhr H, Carli S, Deperschmidt M, Wallwiener D, Zygmunt M, Licht P. Differential effects of human chorionic gonadotropin and decidualization on insulin-like growth factors-I and -II in human endometrial stromal cells. Fertil Steril 2008;90(4 Suppl):1384-9.

19. Fawzy M, El-Refaeey AA. Does combined prednisolone and low molecular weight heparin have a role in unexplained implantation failure? Arch Gynecol Obstet 2014;289:677-80.

20. Qublan H, Amarin Z, Dabbas M, Farraj AE, Beni-Merei Z, Al-Akash $\mathrm{H}$, et al. Low-molecular-weight heparin in the treatment of recurrent IVF-ET failure and thrombophilia: a prospective randomized placebo-controlled trial. Hum Fertil (Camb) 2008;11:246-53.

21. Berker B, Taskin S, Kahraman K, Taskin EA, Atabekoglu C, Sonmezer $\mathrm{M}$. The role of low-molecular-weight heparin in recurrent implantation failure: a prospective, quasi-randomized, controlled study. Fertil Steril 2011;95:2499-502. 